\title{
Sizing of a plastic chamber with air-filled balls for water hammer control
}

\author{
A. Al-Khomairi \& S. Ead \\ Civil Engineering Department, King Saud University, Saudi Arabia
}

\begin{abstract}
Water hammer in pipelines and pipe networks can result in pipe and equipment failures and economical/environmental losses. The use of a piece of flexible plastic pipe for water hammer control is discussed and experimental data for the use of such a device are presented. The device, referred to as a plastic chamber, reduces pressures resulting from water hammer. The flexibility of the plastic chamber allows radial expansion of its walls under pressure to accommodate the excess fluid and thereby reduce water hammer-induced pressure spikes. To enhance the performance of the plastic chamber, air-filled balls are inserted into the chamber. When a sudden pressure increase takes place, the air-filled balls shrink to reduce the pressure surge. Previous experimental data on the use of the plastic chamber and the air-filled balls (inserted in a rigid chamber) are analyzed. The effect of the plastic chamber on the reduction of water hammer was formulated as a function of a dimensionless parameter related to the plastic chamber and the pipe for which water hammer is to be controlled. Furthermore, the effect of the air-filled balls was formulated and found to be a function of an analogous dimensionless parameter related to the volume/inside pressure of the balls and pipe parameters. The two effects were combined in an empirical formula for the total reduction of water hammer when using a plastic chamber with air-filled balls. The procedure allows the required water hammer reducing device to be sized to achieve a target reduction of water hammer based on the parameters of the pipe, the plastic chamber and the air-filled balls.
\end{abstract}

Keywords: unsteady pipe flow, water hammer control, surge pressure control, water hammer damping. 


\section{Introduction}

Water hammer in pipelines and pipe networks can result in huge economical and/or environmental losses. Sudden valve closure, for instance, can generate high-pressure spikes that can damage the pipe and pipe equipment. Several methods have been used to control water hammer and reduce its effect on pipe networks. These include surge vessels, equilibrium tanks, pressure relief valves and suction lines around a booster pump. Other methods used for water hammer control for residential, commercial and light industrial applications include air chambers and water hammer arrestors. An air chamber consists of a short vertical section of pipe that is filled with trapped air. When a water hammer shock occurs, the air chamber acts as a shock absorber. After some time, the water pressure causes the air pocket to be absorbed by water rendering the device ineffective, and the water needs to be drained out of the device. A water hammer arrestor is similar to an air chamber but the air pocket in the arrestor is separated and sealed from the water by a piston with an "O" ring or diaphragm so that the air cannot be absorbed by water. The disadvantage of this device is that the moving piston makes it noisy.

Grundy and Fox [1] suggested the installation of in-line highly deformable tubes to reduce the effect of water hammer. Insertion of a high-density polyethylene (HDPE) polymeric pipe has also been suggested for water hammer suppression in simple pipe system [2]. Pezzinga [3] expanded on the topic by considering a polymeric pipe insertion in a pipe network. Al-Khomairi [4] provided a method of sizing the polymeric pipe insertion for a target water hammer reduction based on the parameters of the pipe and the insertion pipe. Different pipe sizes and insertion parameters/materials were considered. AlKhomairi [5] presented a method using air-filled balls in a steel chamber for water hammer reduction. A steel chamber was used rather than a plastic chamber to isolate the effect of the chamber from the effect of the air-filled balls. This method also allows sizing of the pipe to achieve a target reduction in water hammer.

Al-Khomairi [4] showed that the ability of the polymeric pipe to reduce water hammer is a function of a dimensionless parameter $R_{\mathrm{D}}$ as follows:

$$
R_{\mathrm{D}}=\frac{\Delta V_{\mathrm{D}}}{\Delta V_{\text {max }}}=\frac{1}{2} \frac{\rho a^{2}}{E_{\mathrm{D}}} \frac{D_{\mathrm{D}}}{e_{\mathrm{D}}} \frac{V_{\mathrm{D}}}{V_{\mathrm{P}}},
$$

where $\rho$ is the fluid density, $a$ is the pressure wave velocity in the pipe, $E_{\mathrm{D}}$ is Young's modulus of elasticity for the polymeric pipe insertion, $D_{\mathrm{D}}$ is the diameter of the polymeric pipe insertion, $a_{\mathrm{D}}$ is the pressure wave velocity in the polymeric pipe insertion, $V_{\mathrm{D}}$ is the volume of the polymeric pipe insertion, $V_{\mathrm{P}}$ is the pipe volume and $\Delta \forall_{\mathrm{D}}$ is the increase in volume of the polymeric pipe insertion due to a $\Delta p$ increase in pressure. $\Delta \Psi_{\max }$ is the maximum fluid volume to be admitted for complete elimination of water hammer (during a time interval equal to $2 L / a$ ) and is given by: 


$$
\Delta V_{\max }=Q, \quad t=Q(2 L / a),
$$

where $Q$ is the discharge, $t$ is the time required for the pressure wave to travel from the control valve to hit the boundary and come back to the valve, $L$ is the pipe length and $a$ is as defined earlier.

When using air-filled rubber balls inserted in a rigid steel chamber, this assembly works as a surge pressure absorber. To allow the pressure inside the balls to increase without affecting their volume, the balls are inserted in spherical retaining meshes before being inserted in the chamber. The device can be installed at the location expected to experience the most severe transient event (e.g., just upstream of a control valve) using a $\mathrm{T}$ connector with the pipe. AlKhomairi [5] established a formula that relates the reduction in water hammer when using this device assembly to a function of a dimensionless parameter $R_{\mathrm{B}}$ (analogous to $R_{\mathrm{D}}$ discussed earlier) and is given by:

$$
R_{\mathrm{B}}=\frac{\Delta \forall_{\mathrm{B}}}{\Delta Y_{\text {max }}},
$$

where $\Delta V_{\text {max }}$ is as defined earlier and $\Delta V_{\mathrm{B}}$ is the change in the volume of the ball(s) due to an increase in system pressure. The following equation has been developed by Al-Khomairi [5] to obtain $\Delta V_{\mathrm{B}}$ :

$$
\begin{gathered}
\Delta V_{\mathrm{B}}=\left(h_{\mathrm{atm}}+\Delta h\right) V_{0}\left(\frac{1}{h_{\mathrm{P} 1}+h_{\mathrm{atm}}}-\frac{1}{h_{\mathrm{P} 2}+h_{\mathrm{atm}}}\right), \\
h_{\mathrm{P} 1} \text { and } h_{\mathrm{P} 2}(\text { gage }) \geq \Delta h,
\end{gathered}
$$

where $h_{\text {atm }}$ is the local atmospheric pressure head, $\Delta h$ is the excessive amount of pressure head in the ball $\left(h_{1}-h_{0}\right), h_{1}$ is the gage pressure head inside the ball as pre-determined from the experimental work, $h_{0}$ is the gage pressure head inside the ball when the ball is pressurized until it completely fills the spherical retaining mesh without exerting pressure on it, $V_{0}$ is the volume of the ball associated with $h_{0}, h_{\mathrm{p} 1}$ is the steady-state system hydrostatic pressure head and $h_{\mathrm{p} 2}$ is the pressure head following the spike caused by surge pressure.

The reduction in water hammer-induced pressure surges due to the use of a water hammer absorber is given by:

$$
r=\frac{\Delta h_{\mathrm{wO}}-\Delta h_{\mathrm{W}}}{\Delta h_{\mathrm{wO}}}
$$

where $r$ is the fraction by which water hammer is reduced, $\Delta h_{\mathrm{wo}}$ is the pressure head increase due to water hammer evaluated when no device for water hammer reduction is used and $\Delta h_{\mathrm{W}}$ is the pressure head increase due to water hammer evaluated when the device for water hammer reduction is used to absorb the surge pressure. 
To optimize the performance of the device, the two devices mentioned earlier can be combined by inserting plastic rubber balls (with retaining steel meshes) in a plastic chamber. Both the balls and the plastic chamber contribute to the performance of the device in water hammer mitigation. Thus, the water hammer reduction when using air-filled balls inserted in a plastic chamber is a function of $R_{\mathrm{D}}$ and $R_{\mathrm{B}}$ :

$$
r=f\left(R_{\mathrm{D}}, R_{\mathrm{B}}\right)
$$

\section{Experimental setup}

Two experimental runs were conducted for this study. A single-pipe system consisting of a recirculation tank, upstream pump, pipe, downstream control valve and pressure/flow measurements was used to conduct an extreme transient event experiment. Fig. 1 shows the experimental setup. The experimental work in this study was performed using the same apparatus used by Al-Khomairi [4]. The system and device parameters for the experimental work are: $V$ (fluid velocity) $=2.88 \mathrm{~m} / \mathrm{s}, Q=76 \mathrm{lpm}, D=23.65 \mathrm{~mm}, L=17.23 \mathrm{~m}, D_{\mathrm{D}}=110 \mathrm{~mm}, e_{\mathrm{D}}$ $=3.2 \mathrm{~mm}, L_{\mathrm{D}}$ (chamber length) $=700 \mathrm{~mm}$ and $E_{\mathrm{D}}=3.14 \times 10^{9} \mathrm{~Pa}$. The chamber material used was uPVC. No air-filled balls were inserted in the plastic chamber. Using this experimental setup, fig. 2 shows the variables associated with eqn (5). Fig. 2(a) shows the transient event history following sudden and full closure of a downstream control valve without using a water hammer control device. Fig. 2(b) shows an identical transient event using a plastic chamber for water hammer control. It is clear that a pressure head spike as high as $218 \mathrm{~m}$ without the use of a chamber was reduced to $135 \mathrm{~m}$ when using the device.

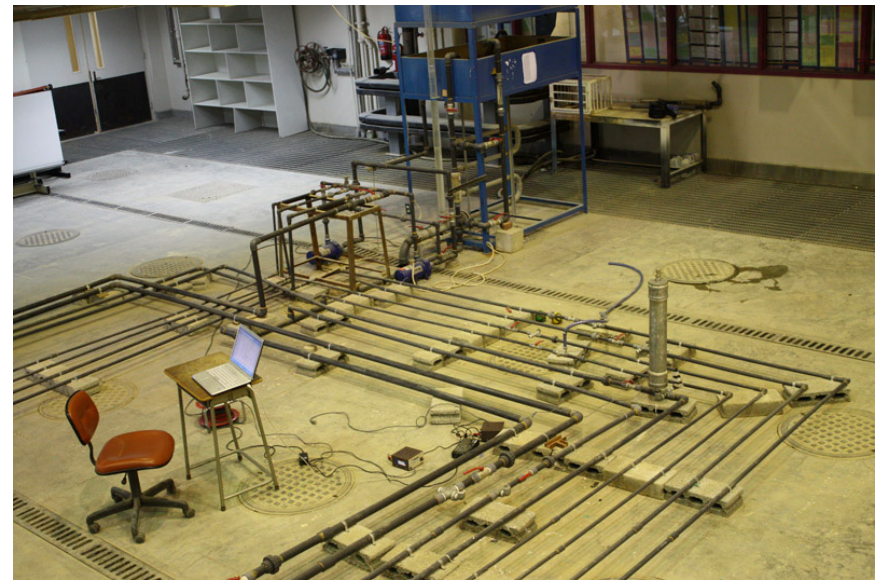

Figure 1: The experimental setup. 

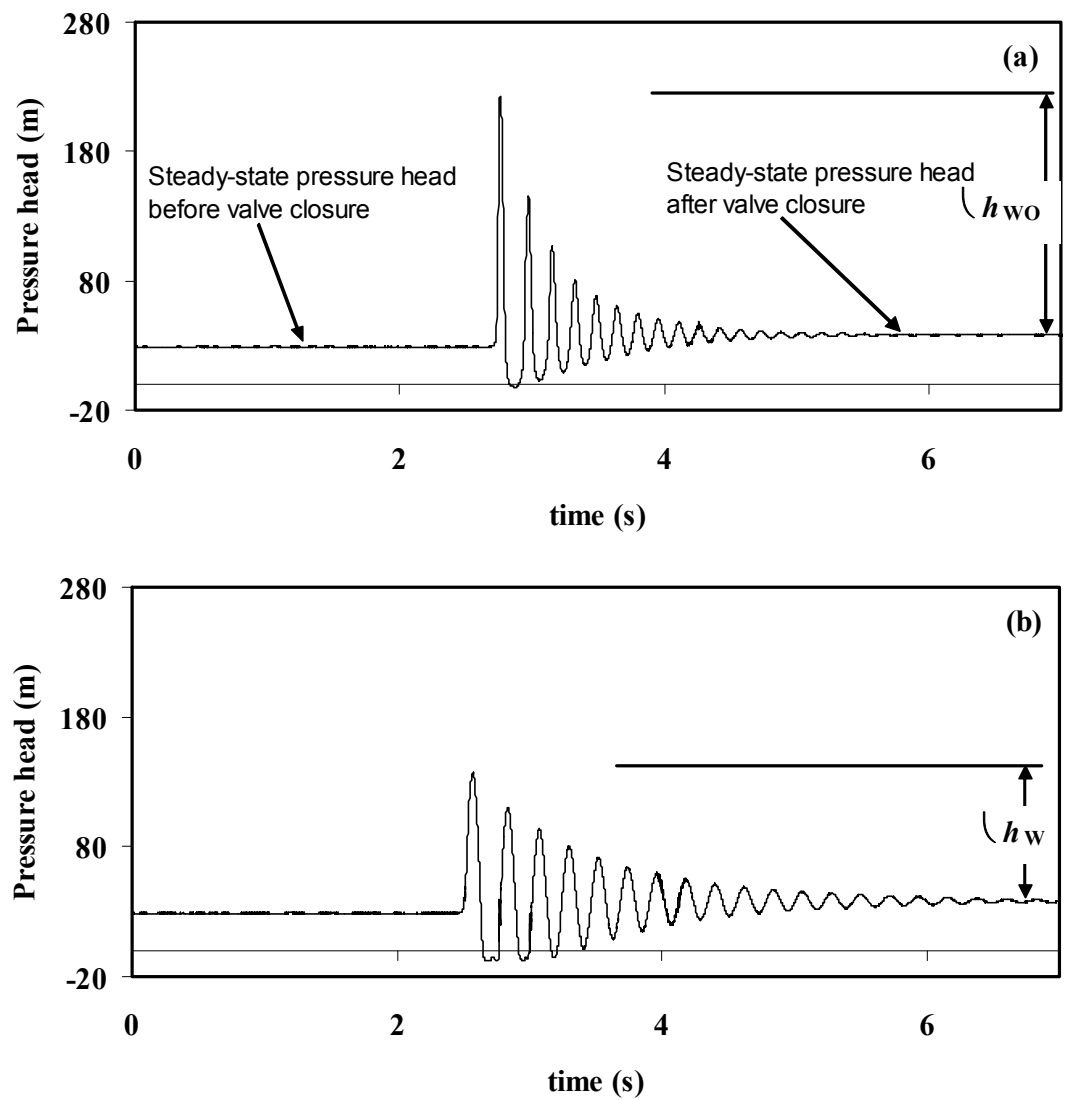

Figure 2: $\quad$ Pressure head history for a severe transient event: (a) without a plastic chamber and (b) with a plastic chamber.

\section{Empirical formula}

The empirical formula that was obtained in this study for the plastic chamber, the air-filled rubber balls and the combined effect of the two systems is explained in this section. Al-Khomairi [4] generated 69 data points using extensive experimental work to relate the ability of the plastic water hammer control device to reduce pressure surges to the dimensionless parameter $R_{\mathrm{D}}$. To generate these data, the plastic chamber and the main pipe parameters were varied and included a wide range of parameter values. An analogous study [5] generated 86 data points relating the performance of the air-filled rubber balls to dimensionless parameters consisting of the relevant pipe and ball parameters. The water hammer reduction in this case was related to the dimensionless 
parameter $R_{\mathrm{B}}$. Inspecting the data for both studies revealed that a logarithmic trend line is the best-fit curved line to represent the data. Using regression analysis the empirical equation for the plastic chamber device is as follows:

$$
r_{\mathrm{D}}=17.997 \ln \left(R_{\mathrm{D}}\right)+44.747,
$$

where $r_{\mathrm{D}}$ is the $\%$ reduction in water hammer when using the plastic chamber for water hammer control and $R_{\mathrm{D}}$ is the dimensionless parameter associated with the chamber and the pipe as revealed in eqn (1).

By analogy, using regression analysis for air-filled balls placed in a rigid (steel) chamber, the following empirical equation is achieved for the device performance:

$$
r_{\mathrm{B}}=11.488 \ln \left(R_{\mathrm{B}}\right)+62.968,
$$

where $r_{\mathrm{B}}$ is the $\%$ reduction in water hammer when the device with air-filled balls is used to control water hammer and $R_{\mathrm{B}}$ is a dimensionless parameter representing the balls and pipe parameters as shown in eqn (3). Figs. 3 and 4 depict the resulting regression curves for the plastic chamber and the air-filled balls, respectively. It is logical that for both curves, with increased $R_{\mathrm{B}}$ or $R_{\mathrm{D}}$, the reduction in water hammer approaches $100 \%$.

It is possible to predict the performance of air-filled balls installed in a plastic chamber by combining the outcome of the two studies. The following equation can be used to find the combined reduction for the air-filled balls inserted in a plastic air chamber:

$$
r_{\mathrm{T}}=\left(1-\left(1-0.01 r_{\mathrm{D}}\right)\left(1-0.01 r_{\mathrm{B}}\right)\right) \times 100,
$$

where $r_{\mathrm{T}}$ is the total $\%$ reduction using the device that combines air-filled balls with a plastic chamber.

For example, a reduction of $30 \%$ by the plastic chamber and $40 \%$ by the airfilled balls means a reduction of $58 \%$ if the air-filled balls are placed in the plastic chamber.

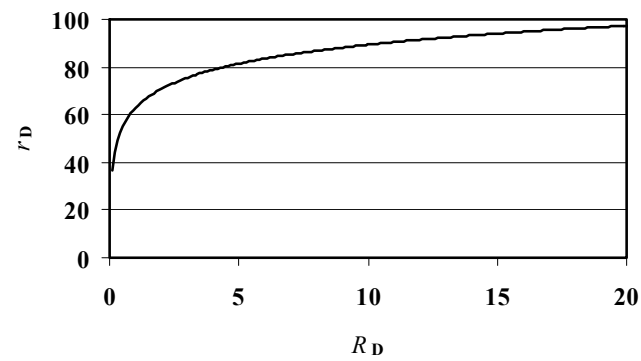

Figure 3: $\quad$ Pipe and plastic chamber: dimensionless parameter $\left(R_{\mathrm{D}}\right)$ versus $\%$ reduction $\left(r_{\mathrm{D}}\right)$ in water hammer-induced pressure surge. 


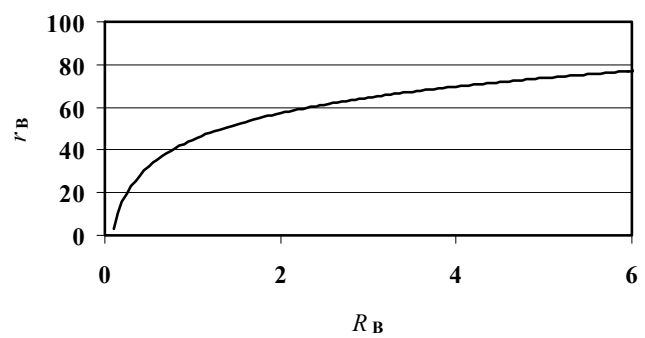

Figure 4: $\quad$ Pipe and air-filled balls: dimensionless parameter $\left(R_{\mathrm{B}}\right)$ versus \% reduction $\left(r_{\mathrm{B}}\right)$ in water hammer-induced pressure surge.

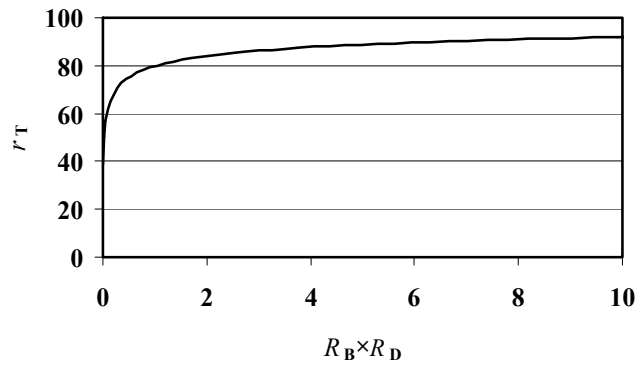

Figure 5: Percentage reduction in water hammer $\left(r_{\mathrm{T}}\right)$ due to the combined effect of air-filled balls $\left(R_{\mathrm{B}}\right)$ and the plastic chamber $\left(R_{\mathrm{D}}\right)$.

Plotting the product of the two variables, $R_{\mathrm{D}} \times R_{\mathrm{B}}$ versus the total reduction $\left(r_{\mathrm{T}}\right)$ provides a relationship between the parameters of the plastic chamber, airfilled balls (and pipe) and the total \% reduction achieved by the device with the plastic chamber and the air-filled balls. Fig. 5 was obtained using the data from fig. 3, fig. 4 and eqn (9). The $r_{\mathrm{T}}$ axis scale was limited to a value of 10 to show a clearer picture of how the reduction changes for low $R_{\mathrm{D}} \times R_{\mathrm{B}}$ values. Fig. 5 can be used to predict the performance of the water hammer reducing device when air-filled balls are inserted in the plastic chamber. For example, computation of $R_{\mathrm{D}}$ and $R_{\mathrm{B}}$ based on the parameters of the pipe, plastic chamber and the balls allows one to use the curve to read the corresponding \% reduction $\left(r_{\mathrm{D}}\right)$ for the device. Furthermore, it is possible to design a device with a plastic chamber and air-filled balls for a target $\%$ total reduction $\left(r_{\mathrm{T}}\right)$ value. From fig. 5 and a target $r_{\mathrm{T}}$ value, the corresponding $R_{\mathrm{D}} \times R_{\mathrm{B}}$ value can be read from the graph. The values of the parameter(s) that give the same $R_{\mathrm{D}} \times R_{\mathrm{B}}$ value can be selected. For example, if the parameters for the plastic chamber are fixed because of space limitations, the ball volume/pressure that would result in the required $R_{\mathrm{D}} \times R_{\mathrm{B}}$ value can be selected. It is also possible to use eqns (7), (8) and (9) instead of fig. 5 to determine the required parameters. If a plastic chamber is used without airfilled balls, eqn (7) or fig. 3 can be used to determine the design parameters. 
However, eqn (8) or fig. 4 can be used for the analysis/design if air-filled balls are used in a rigid (e.g., steel) chamber for water hammer control.

\section{Conclusions}

The results of water hammer control in pipelines using two different methods are presented and analyzed. The first method uses a plastic chamber with a diameter much larger than the pipe for which water hammer is to be controlled. In the second method, air-filled rubber balls are inserted into a rigid (steel) chamber. Laboratory tests performed in earlier studies for both methods were analyzed. Regression analysis was performed on the data to achieve the most accurate empirical formula to determine the ability of each device to reduce water hammer as a function of dimensionless parameters related to the pipe, balls and plastic chamber.

A more effective method for water hammer mitigation would be to combine the effect of the two methods by inserting the air-filled rubber balls into a plastic chamber. An empirical formula was devised for the combined effect of the two methods on water hammer reduction. Equations and figures were established for each method and for the combined method to analyze device performance based on given pipe, balls and chamber pipe parameters. A target water hammer reduction can be achieved using these formula and graphs to design the device.

\section{References}

[1] Grundy, A.K. \& Fox, J.A. The effect of compressible pipeliners on pressure transient generation. Proceedings of the 3rd International Conference on Pressure Surges. BHRA: Cranfield, UK, pp. 241-260, 1980.

[2] Pezzinga, G. \& Scandura, P. Unsteady flow in installation with polymeric additional pipe. Journal of Hydraulic Engineering 121(11), pp, 802-811, 1995.

[3] Pezzinga, G. Unsteady flow in hydraulic networks with polymeric additional pipe. Journal of Hydraulic Engineering 128(2), pp. 238-244, 2002.

[4] Al-Khomairi, A.M. Plastic water hammer damper. Journal of Pipeline Systems Engineering and Practice (submitted for publication).

[5] Al-Khomairi, A.M. Surge pressure suppression in pipelines using air-filled balls. Journal of King Abdulaziz University (submitted for publication). 